\title{
Bone Marrow Derived Kit-positive Cells Colonize the Gut but Fail to Restore Pacemaker Function in Intestines Lacking Interstitial Cells of Cajal
}

\author{
Conor J McCann, ${ }^{1,2}$ Sung-Jin Hwang, ${ }^{1}$ Grant W Hennig, ${ }^{1}$ Sean M Ward ${ }^{1}$ and Kenton M Sanders ${ }^{1 *}$ \\ ${ }^{1}$ Department of Physiology and Cell Biology, University of Nevada School of Medicine, Reno, NV, USA; and ${ }^{2}$ University College London Institute \\ of Child Health, Birth Defects Research Center, Neural Development Unit, London, UK
}

\begin{abstract}
Background/Aims
Several motility disorders are associated with disruption of interstitial cells of Cajal (ICC), which provide important functions, such as pacemaker activity, mediation of neural inputs and responses to stretch in the gastrointestinal (GI) tract. Restoration of ICC networks may be therapeutic for GI motor disorders. Recent reports have suggested that Kit ${ }^{+}$cells can be restored to the GI tract via bone marrow (BM) transplantation. We tested whether BM derived cells can lead to generation of functional activity in intestines naturally lacking ICC.
\end{abstract}

\section{Methods}

BM cells from Kit ${ }^{+/ c o p G F P}$ mice, in which ICC are labeled with a green fluorescent protein, were transplanted into W/WV intestines, lacking ICC. After 12 weeks the presence of ICC was analyzed by immunohistochemistry and functional analysis of electrical behavior and contractile properties.

Results

After 12 weeks copGFP ${ }^{+}$BM derived cells were found within the myenteric region of intestines from $W / W^{V}$ mice, typically populated by ICC. Kit cells failed to develop interconnections typical of ICC in the myenteric plexus. The presence of Kit ${ }^{+}$cells $^{+}$ was verified with Western analysis. BM cells failed to populate the region of the deep muscular plexus where normal ICC density, associated with the deep muscular plexus, is found in W/W mice. Engraftment of $\mathrm{Kit}^{+}-\mathrm{BM}$ cells resulted in the development of unitary potentials in transplanted muscles, but slow wave activity failed to develop. Motility analysis showed that in testinal movements in transplanted animals were abnormal and similar to untransplanted $W / W^{V}$ intestines.

\section{Conclusions}

$\mathrm{BM}$ derived $\mathrm{Kit}^{+}$cells colonized the gut after BM transplantation, however these cells failed to develop the morphology and function of mature ICC.

(J Neurogastroenterol Motil 2014;20:326-337)

Key Words

Bone marrow transplantation; Electrophysiology; Interstitial cells of Cajal

Received: March 5, 2014 Revised: April 1, 2014 Accepted: April 9, 2014

(c) This is an Open Access article distributed under the terms of the Creative Commons Attribution Non-Commercial License (http://creativecommons. org/licenses/by-nc/3.0) which permits unrestricted non-commercial use, distribution, and reproduction in any medium, provided the original work is properly cited.

*Correspondence: Kenton M Sanders, PhD

Department of Physiology and Cell Biology, University of Nevada School of Medicine, Reno, NV 89557 USA

Tel: +1-775-784-6908, Fax: +1-775-784-6903, E-mail: ksanders@medicine.nevada.edu

Financial support: This work was supported by a program project grant (Grant No. NIH P01 DK41315). Morphological studies were performed in a Core laboratory supported by NIH P01 DK41315. Confocal images were collected using a Zeiss LSM510 Meta confocal microscope obtained with support from NIH1 S10 RR16871.

Conflicts of interest: None.

Author contributions: Conor J McCann conducted experiments, performed data analysis and drafted manuscript; Sung-Jin Hwang and Grant W Hennig conducted experiments and performed data analysis; Sean M Ward designed experiments and drafted manuscript. Kenton M Sanders designed experiments, drafted the manuscript and supervised the study.

ORCID: Conor J McCann, http://orcid.org/0000-0002-9324-5984; Sung-Jin Hwang, http://orcid.org/0000-0002-7571-7650; Grant W Hennig, http://orcid.org/0000-0001-7436-9458; Sean M Ward, http://orcid.org/0000-0003-0217-1535; Kenton M Sanders, http://orcid.org/00000002-4196-1583. 


\section{Introduction}

Loss of interstitial cells of Cajal (ICC) or disruption of ICC networks have been implicated in a variety of GI motility disorders including achalasia, ${ }^{1}$ slow transit constipation, ${ }^{2}$ intestinal pseudoobstruction, ${ }^{3}$ Crohn's disease, ${ }^{4}$ inflammation ${ }^{5}$ and diabetic gastroparesis. ${ }^{6,7}$ Although ICC defects have been associated with these disorders, there have been few studies to determine whether ICC can be made to repopulate gastrointestinal (GI) muscles with the goal of recovering normal motor activity.

ICC arise from mesenchymal precursors, ${ }^{8,9}$ however the mechanisms of post-natal ICC turnover and maintenance of ICC populations remain unclear. In animal models ICC have been shown to redifferentiate toward a smooth muscle phenotype following a GI insult. ${ }^{10,11}$ It has also been reported that ICC can undergo mitosis and/or apoptosis to regulate cell numbers during normal homeostasis. ${ }^{12}$ Others have suggested the presence of a stem cell population that is capable of regeneration of ICC. ${ }^{13}$

In neonatal tissues ICC demonstrate remarkable plasticity and capacity for regeneration as normal networks and functionality can be re-established after Kit signaling is blocked and ICC networks are disrupted. ${ }^{14}$ In some instances regeneration has also been demonstrated in adult muscles after pathophysiological conditions have caused disruption of ICC networks, ${ }^{15,16}$ however how this happens and whether it is a generalized capability of ICC in adult animals is not clear. Bone marrow (BM) derived stem cells offer a promising source of pluripotent regenerative cells which might provide a mechanism of ICC regeneration, and mesenchymal stem cells have been shown to repopulate cardiac, ${ }^{17,18}$ hepatic, ${ }^{19,20}$ pancreatic $^{21}$ and lung ${ }^{22}$ tissues after BM transplantation. Recently, $\mathrm{Kit}^{+} \mathrm{BM}$ derived cells have been shown to repopulate the small intestine in response to intestinal injury $^{23}$ and BM transplantation has been reported to increase GI transit in $W / W^{V}$ mice in which specific populations of ICC fail to develop and pacemaker activity is compromised. ${ }^{24}$ These findings suggest that BM transplantation may provide therapeutic interventions in patients with ICC loss and dysmotility.

We investigated whether BM transplantation from mice with normal ICC networks and pacemaker activity would allow development of: (1) $\mathrm{Kit}^{+}$ICC networks and (2) pacemaker activity in the small intestines of $W / W^{V}$ mice with congenital electrical quiescence. We found that $\mathrm{Kit}^{+}$cells derived from BM tracked to the gut and repopulated the region of the myenteric plexus normally populated by pacemaker ICC (ICC-MY). These cells displayed some of the characteristics of typical ICC however, they failed to develop into networks or develop the ability to generate electrical slow waves. Thus, BM transplantation provides a method of delivering $\mathrm{Kit}^{+}$cells but other tissue signals, possibly lacking in the $W / W^{V}$ intestine, appear to be required for BM derived cells to develop into functional ICC networks.

\section{Materials and Methods}

\section{Animals}

Animals used for these studies were maintained and the experiments performed in accordance with the National Institutes of Health Guide for the Care and Use of Laboratory Animals. The Institutional Animal Use and Care Committee at the University of Nevada approved all procedures used.

\section{Bone Marrow Cell Preparation}

$\mathrm{BM}$ was isolated as previously described. ${ }^{25}$ Briefly, donor C57BL/6 (Jackson Laboratory, Bar Harbor, MN, USA) and $\mathrm{Kit}^{+\operatorname{cop} G F P}$ (Generated at the University of Nevada, Reno, USA) animals were euthanized via administration of $\mathrm{CO}_{2}$. The spine, fibulae and tibiae were removed to phosphate buffered saline (PBS) containing 1\% anitibiotic-antimycotic (Gibco, Grand Island, NY, USA). Bone marrow cell (BMC) suspensions were prepared by gently releasing the cells with a pestle and mortar into PBS. The cells were filtered through a polyester filter with 30 $\mu \mathrm{m}$ mesh size (Miltenyi Biotec, Auburn, CA, USA) to remove particulates, washed twice and resuspended to the appropriate concentration in PBS $\left(1 \times 10^{7}\right.$ cells $\left./ 500 \mu \mathrm{L}\right)$ for transplantation.

\section{Bone Marrow Cell Transplantation}

BM transplantation was performed as previously described. ${ }^{25}$ Briefly, donor recipient $W / W^{V}$ mice (Jackson Laboratory, Bar Harbor, MN, USA) were housed in specific pathogen-free conditions throughout and treated with antibiotics $(32 \mathrm{~mL}$ Sulfatrim per liter of de-ionized drinking water; Actavis, Baltimore, MD, USA) for 10 days prior and 2 weeks post irradiation. Recipient mice received $9 \mathrm{~Gy}$ total body irradiation from a ${ }^{137} \mathrm{C}$ s source followed by intravenous infusion of donor BMCs via tail vein injection. All experiments were carried out at 12 weeks post transplantation.

\section{Electrophysiological Experiments}

Small intestines were removed after animals were euthanized 
following sedation with isoflurane and cervical dislocation. Tissues were placed in oxygenated cold $\left(4^{\circ} \mathrm{C}\right) \mathrm{KRB}$ for further preparation.

After fine dissection of the mucosa and submucosa small preparations (approximately $10 \mathrm{~mm}^{2}$ ) were pinned, with the luminal side of the circular muscle up, to Sylgard elastomer-coated bases of $35 \mathrm{~mm}$ polypropylene dishes (Corning Glass Works, Corning, NY, USA).

Cells were impaled with glass microelectrodes filled with 3 $\mathrm{M} \mathrm{KCl}$ and having resistances between 80 and $120 \mathrm{M} \Omega$. Transmembrane potentials were measured using a high input impedance amplifier (Axon Instruments/Molecular Devices Corp., Sunnyvale, CA, USA) and outputs displayed on a digital oscilloscope. Electrical signals were digitized using an analog-to-digital converter (Digidata 1300 series; Axon Instruments), recorded and stored on a computer running Axoscope 9.0 software. Electrical recordings were made in the presence of nifedipine $(1 \mu \mathrm{M})$ to reduce muscle contraction and maintain cellular impalements.

\section{Western Blot Analysis}

Total protein was extracted from control and BM recipient small intestine. Protein $(30 \mu \mathrm{g})$ was subjected to electrophoresis on $10 \%$ SDS-polyacrylamide gel (SDS-PAGE), and transferred onto nitrocellulose membranes (Bio-Rad Laboratories, CA, USA). Goat anti-Kit (1:500; R\&D Systems, Minneapolis, MN, USA) and rabbit anti-GAPDH (1:2000; Santa Cruz Biotechnology, Santa Cruz, CA, USA) primary antibodies were used. Protein analysis was performed using Quantity One 4.5.1 software (Bio-Rad Laboratories) and Image-J software (NIH, Bethesda, MD, USA) was used to calculate the relative densities of Kit protein compared with housekeeper glyceraldehyde-3phosphate dehydrogenase (GAPDH). Control experiments were performed in the absence of primary or secondary antibodies.

\section{Immunohistochemistry}

Tissues were fixed in paraformaldehyde $(4 \% \mathrm{w} / \mathrm{v}$ in $0.1 \mathrm{M}$ PBS for 15 minutes at room temperature). After fixation, the tissues were washed for 24 hours, with multiple changes in PBS (0.01 M, pH 7.2) and blocked for 1 hour at room temperature with bovine serum albumin ( $1 \% \mathrm{w} / \mathrm{v}$; Sigma-Aldrich, St. Louis, MO, USA). Tissues were incubated in primary antibodies for 48 hours at $4^{\circ} \mathrm{C}$. ICC, mast cells and macrophages were identified with goat polyclonal anti-mouse stem cell factor receptor (2 $\mu \mathrm{g} / \mathrm{mL}$; R\&D Systems), rabbit polyclonal anti-synthetic hista- mine ( $2 \mu \mathrm{g} / \mathrm{mL}$; Immunostar, Hudson, WI, USA) and rat monoclonal anti-mouse F4/80 (2 $\mu \mathrm{g} / \mathrm{mL}$; Seroted, Oxford, England), respectively, in $0.01 \mathrm{M}$ PBS containing $0.5 \%$ Triton-X 100. Immunoreactivity was detected using Alexa Fluor 594 donkey anti-goat secondary antibody, Alexa Fluor 488 sheep anti-rabbit and Alexa Fluor 488 donkey anti-rat (1:1000 in PBS, 1 hour, room temperature; Invitrogen, Carlsbad, CA, USA).

Before mounting, tissues were thoroughly washed (0.01 M PBS for 16 hours) to remove excess secondary antibody. Control experiments were performed in the absence of primary or secondary antibodies. Mounted specimens were examined using Zeiss LSM510 Meta (Carl Zeiss, Jena, Germany). Confocal micrographs of whole mounts were digital composites of the Z-series of scans of $0.5 \mu \mathrm{m}$ optical sections. Final images were constructed using LSM image browser (Carl Zeiss) and Image-J software (NIH).

\section{Surface Marker Array of Contraction Coordination}

Segments of ileum approximately $3-5 \mathrm{~cm}$ in length were pinned in a Sylgard dish containing oxygenated Krebs' solution at room temperature. The preparation was opened along the mesenteric border, pinned as a flat-sheet and the mucosa and submucosa removed by sharp dissection. Surface markers were positioned on the topmost surface in a grid $(3$ circumferential $\times 6-9$ longitudinal). The tissue was then repinned so that only the oral, anal and middle regions were pinned to allow for greater movement. Longitudinal movement was used instead of circular movement due to the much larger contractions. Circular movements were contaminated by the much stronger longitudinal contractions.

The movements of the preparation were recorded by video (DMK 31AF03; Imaging Source, Charlotte, NC, USA) onto a computer (iMac; Apple Inc., Cupertino, CA, USA), then imported into custom-written software (Volumetry G7mv; written by Grant W. Hennig) where the position of surface markers and edges of the preparation were tracked. Spatio-temporal maps (STMaps) of longitudinal movements were constructed by measuring the distance between pairs on markers in the longitudinal axis, converting the distance to a grayscale value. ${ }^{26}$ Linear interpolation between 2 grayscale values was used to fill in spaces between surface markers. The grayscale values were scaled in the following manner to compensate for slight variations in the distances between pairs of markers. The average longitudinal distance between each pair of markers for the duration of the movie 


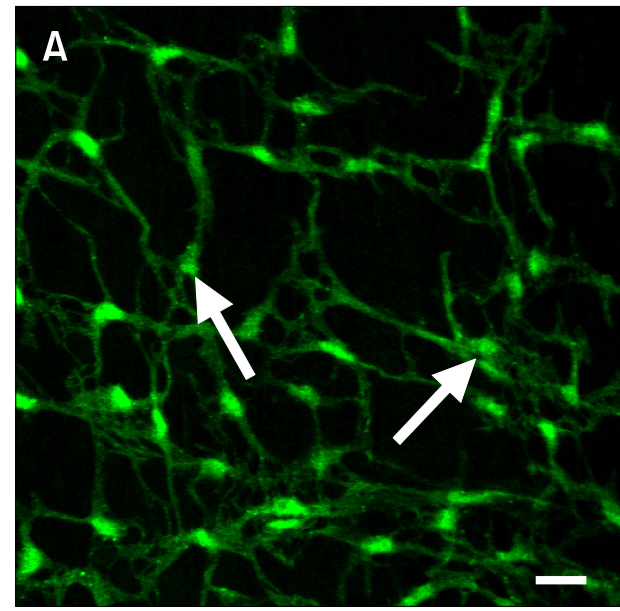

B

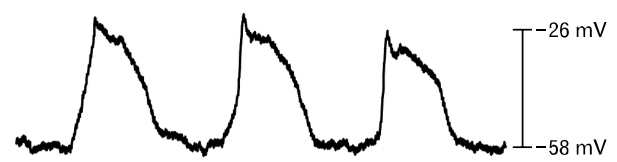

D

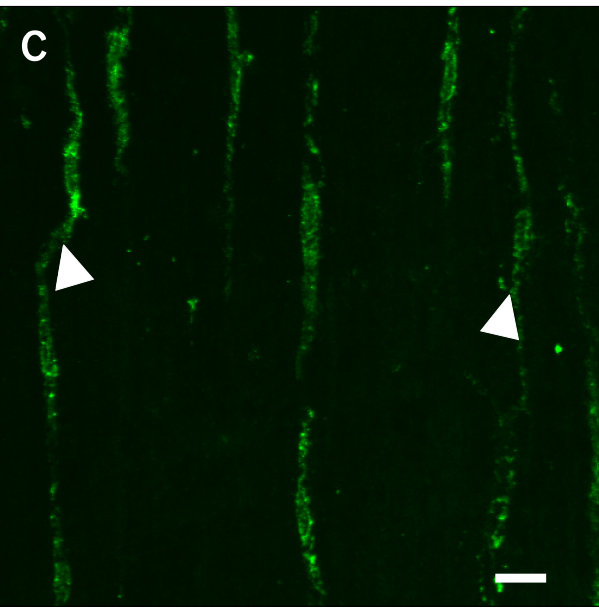

Figure 1. Presence or absence of interstitial cells of Cajal (ICC)-networks and pacemaker activity in the small intestines of $\mathrm{Kit}^{+{ }^{+} \text {copGFP }}$ and $W / W^{V}$ mice. The small intestines of $\mathrm{Kit}^{+{ }^{+} \operatorname{cop} G F P}$ mice display normal myenteric ICC (ICC-MY) networks (A, arrows) and slow wave activity (B). Loss of ICC-MY, but not ICC associated with the deep muscular plexus (arrowheads) in $W / W^{V}$ small intestines (C) is associated with an absence of slow waves (D). Scale bars $=20 \mu \mathrm{m}$.
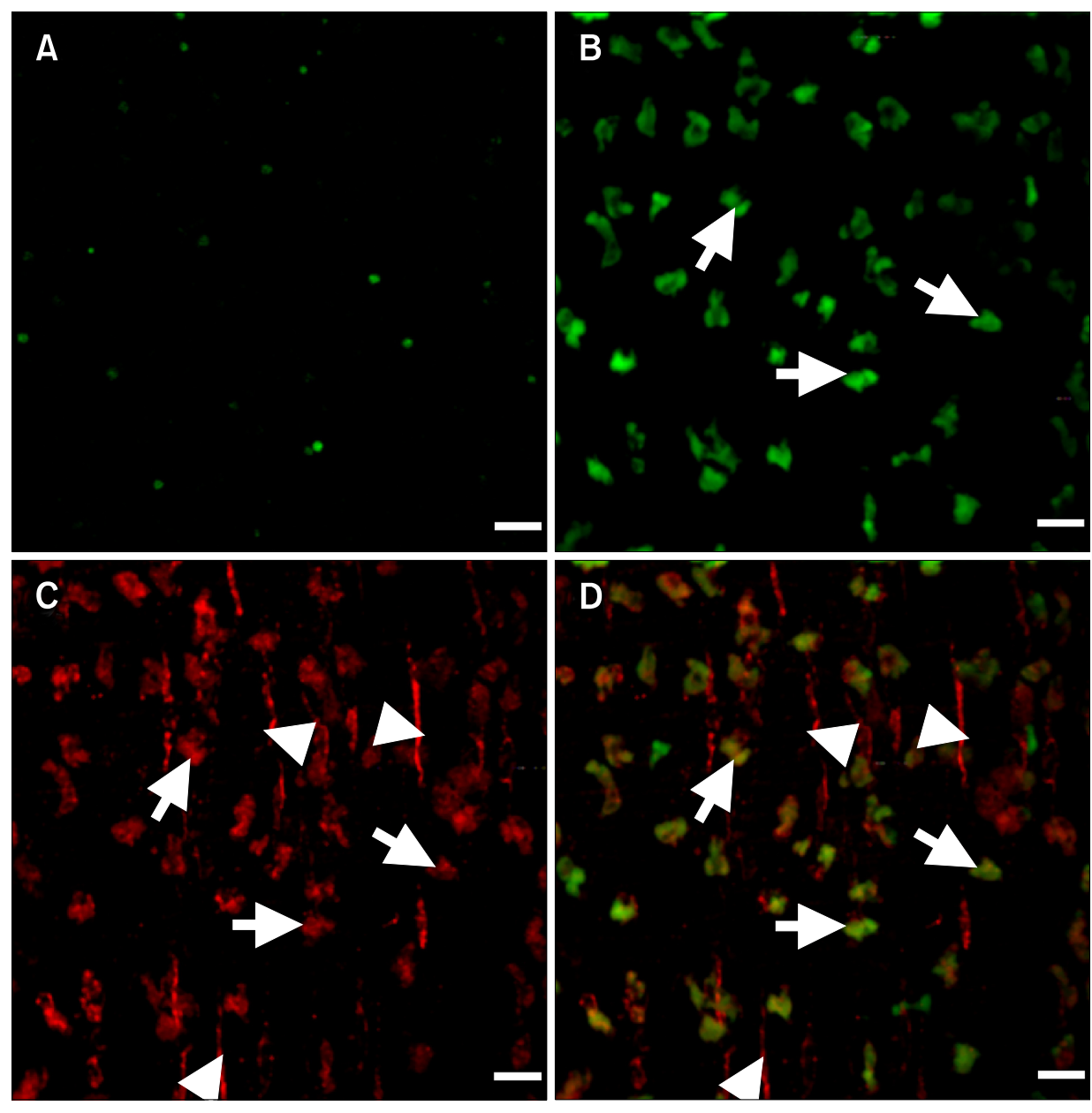

Figure 2. Transplantation leads to the formation of clusters of bone marrow (BM) derived interstitial cells of Cajal (ICC) at the level of the myenteric plexus. (A) Confocal micrograph demonstrating expression of copGFP in $\mathrm{BM}$ cells from $\mathrm{Kit}^{+/ c o p G F P}$ mice. (B) Shows presence of $\mathrm{GFP}^{+} \mathrm{BM}$ derived cells at the level of the myenteric plexus (arrows) in transplanted $W / W^{V}$ intestines (C) Kit immunoreactivity highlighting the presence of $\mathrm{Kit}^{+} \mathrm{ICC}$ at the level of the myenteric plexus (arrows) and deep muscular plexus (arrowheads). (D) Merged image highlighting the co-labeling of $\mathrm{GFP}^{+} \mathrm{BM}$ derived cells and Kit at the level of the myenteric plexus (arrows), note ICC associated with the deep muscular plexus (arrowheads) stain only for Kit. Scale bars $=20 \mu \mathrm{m}$. 
was calculated, then a scaling factor calculated so that the average distance equalled $1 \mathrm{~mm}$ (e.g., if the average distance between markers was $0.75 \mathrm{~mm}$, a scaling factor of 1.33 was used). The average distance was assigned mid-level gray on the STMap. Movements of pairs of markers closer together (contraction whiter values), or further apart (elongation - darker values) were scaled using their respective scaling factor. The unit of measurement is $\Delta \mathrm{mm}$ per $1 \mathrm{~mm}$ average separation $(\Delta \mathrm{mm} / \mathrm{mm})$.

\section{Solutions and Drugs}

The electrophysiological bath chamber was constantly perfused with oxygenated Krebs-Ringer buffer (KRB) of the following composition (mM): $\mathrm{NaCl} 118.5 ; \mathrm{KCl} 4.5 ; \mathrm{MgCl}_{2} 1.2$; $\mathrm{NaHCO}_{3}$ 23.8; $\mathrm{KH}_{2} \mathrm{PO}_{4}$ 1.2; dextrose 11.0; and $\mathrm{CaCl}_{2}$ 2.4. The $\mathrm{pH}$ of the $\mathrm{KRB}$ was 7.3-7.4 when bubbled with $97 \% \mathrm{O}_{2}-3 \% \mathrm{CO}_{2}$ at $37 \pm 0.5^{\circ} \mathrm{C}$. Muscles were left to equilibrate for at least 1 hour prior to impalement. For electrophysiological experiments nifedipine (Sigma; St Louis, MO, USA) was dissolved in ethanol at a stock concentration of $100 \mu \mathrm{M}$ before being added to the perfusion solution at a final concentration of $1 \mu \mathrm{M}$ to inhibit contractile activity. It has been previously reported that nifedipine (1 $\mu \mathrm{M}$ ) does not affect small intestinal slow wave activity. ${ }^{27}$

\section{Results}

\section{Development of $\mathrm{Kit}^{+}$Cells in Bone Marrow Transplanted Intestines}

Small intestinal muscles of $W / W^{V}$ mice lack most ICC-MY and fail to develop normal pacemaker activity that drives segmental contractions (Fig. 1). ${ }^{27,28}$ The only known genetic defect in $W / W^{V}$ mice is compromised Kit signaling, so these mice provide a useful model to determine whether cells with normal Kit alleles can successfully target, engraft and develop normal function in intestinal muscles. The ability of $\mathrm{BM}$ derived $\mathrm{Kit}^{+}$cells to repopulate appropriate niches within the intestinal wall and establish pacemaker activity was investigated using BMCs from transgenic $\mathrm{Kit}^{+\operatorname{cop} G F P}$ mice that express a bright green fluorescent protein driven by the endogenous, cell-specific Kit promoter. ${ }^{29}$ BM cells (Fig. 2A) were harvested from these mice and injected into $W / W^{V}$ mice, and characterization of BM derivatives in intestinal muscles was performed after 12 weeks. A period of 12 weeks was chosen for the developmental period because this is $50 \%$ longer than required for re-establishment of ICC networks in tissues made devoid of ICC-MY by bowel obstruction. ${ }^{16}$ A similar time scale has also been used in other investigations of BM extravasation to GI tissues. ${ }^{23,30}$ Control experiments were also performed using BM cells derived from a $\mathrm{C} 57 \mathrm{BL} / 6$ strain injected into $W / W^{V}$ mice.

Examination of intestinal tissues from $W / W^{V}$ mice 12 weeks after BM transplantation revealed engraftment and development of cop $\mathrm{GFP}^{+}$cells in the small intestine and specifically at the level of the myenteric plexus (Fig. 2B). Cells with copGFP ${ }^{+}$displayed a triangular morphology reminiscent of cell bodies of ICC-MY in wild type intestinal muscles. However, cellular processes, also typical of ICC-MY, were not observed (Fig. 2). Double labeling confirmed that $\mathrm{BM}$-derived copGFP ${ }^{+}$cells in the intestine expressed Kit-like immunoreactivity (Fig. 2C and 2D). $\mathrm{Kit}^{+} \mathrm{ICC}$ develop in the region of the deep muscular plexus (ICC-DMP) in the small intestines of $W / W^{V}$ mutants. ${ }^{27}$ Hence we also examined this region to determine if cells with copGFP engrafted in a region already populated with ICC. We confirmed
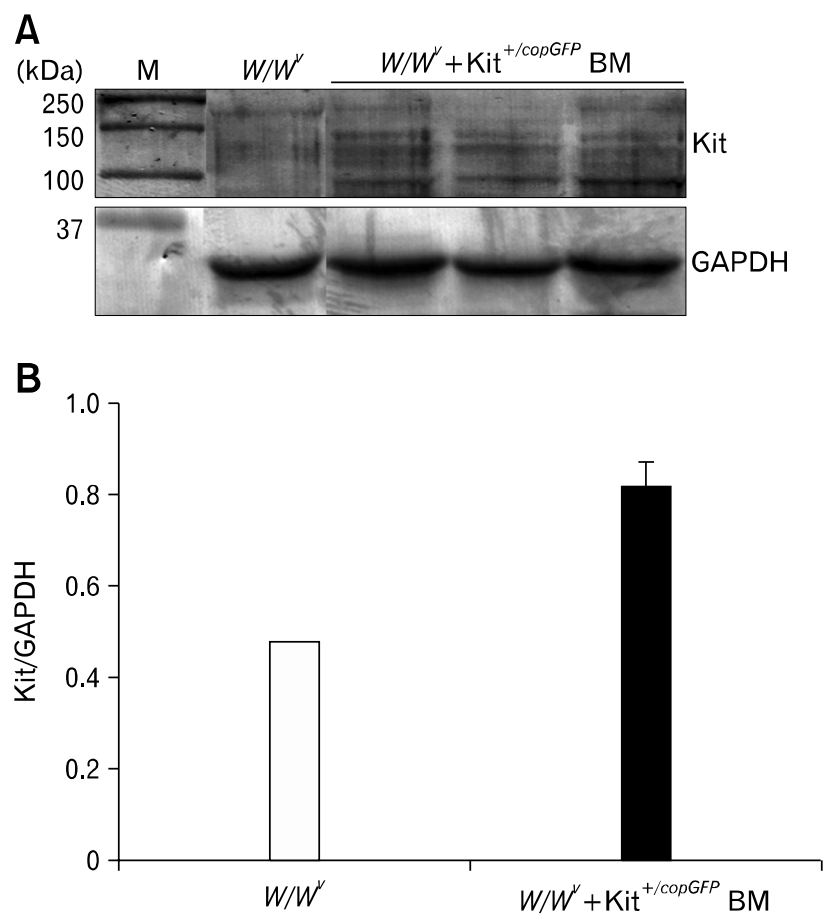

Figure 3. Quantification of the increased Kit protein expression in transplanted $W / W^{V}$ tissues. (A) Development of bone marrow (BM) derived interstitial cells of Cajal is mirrored by an increase in total Kit protein expression when analyzed via Western analysis. (B) BM transplantation of $\mathrm{Kit}^{+{ }^{+} \text {cop GFP }}$ led to a 1.68-fold increase in Kit expression with the small intestines of transplanted $W / W^{V}$ tissue. Tissues were normalized to glyceraldehyde-3-phosphate dehydrogenase (GAPDH) expression. 
the presence of $\mathrm{Kit}^{+}$ICC-DMP in $W / W^{V}$ mice, but BM derived cells with copGFP ${ }^{+}$were rarely observed in the DMP (Fig. 2D).

Changes in Kit expression were quantified in $W / W^{V}$ mice transplanted with BM derived $\mathrm{Kit}^{+/ \operatorname{cop} G F P}$ cells by Western analysis performed on extracts of tunica muscularis collected from the same animals used for immunohistochemical studies. Development of cells with copGFP in the myenteric plexus region was associated with an increase in total Kit protein expression (Fig. 3). Although Kit is driven from only one allele in $\mathrm{Kit}^{+/ \operatorname{cop} G F P}$ mice, development of $\mathrm{Kit}^{+}$cells with copGFP in transplanted $W / W^{V}$ mice led to a $1.68 \pm 0.13(P=0.008, \mathrm{n}=3)$ fold increase in total Kit protein expression in comparison to non-transplanted $W / W^{V}$ muscles (Fig. 3).

We investigated whether BM derived cells with copGFP found in the myenteric plexus region after transplantation were other types of cells of hematopoietic origin commonly found in the tunica muscularis (i.e., mast cells or macrophages) by double labeling with antibodies against histamine or $\mathrm{F} 4 / 80 .{ }^{31} \mathrm{Kit}^{+}$cells within the myenteric plexus region were found to be negative for histamine and F4/80 (Fig. 4), suggesting that the copGFP ${ }^{+} / \mathrm{Kit}^{+}$ cells that develop after BM transplantation are likely to be ICC that fail to develop a fully mature phenotype (Fig. 4).

\section{Intestinal Electrical Activity Following Bone Marrow Transplantation}

Since the goal of these studies was to determine whether functional ICC could develop after BM transplantation, we also characterized the electrical activity of intestinal circular muscle cells from control, non-transplanted $W / W^{V}$ intestines and BM transplanted intestinal muscles. Control muscles displayed resting membrane potentials (RMP) averaging $-52.8 \pm 1.0 \mathrm{mV}$ and were electrically quiescent (Fig. 1D and $5 ; \mathrm{n}=4$ ), as previously reported. ${ }^{27,28}$ RMPs of $W / W^{V}$ intestines transplanted with $\mathrm{BMCs}$ from $\mathrm{Kit}^{+/ \operatorname{cop} G F P}$ mice were significantly more negative, averaging $-59.0 \pm 0.4 \mathrm{mV}(P=0.001, \mathrm{n}=4)$. Similarly,
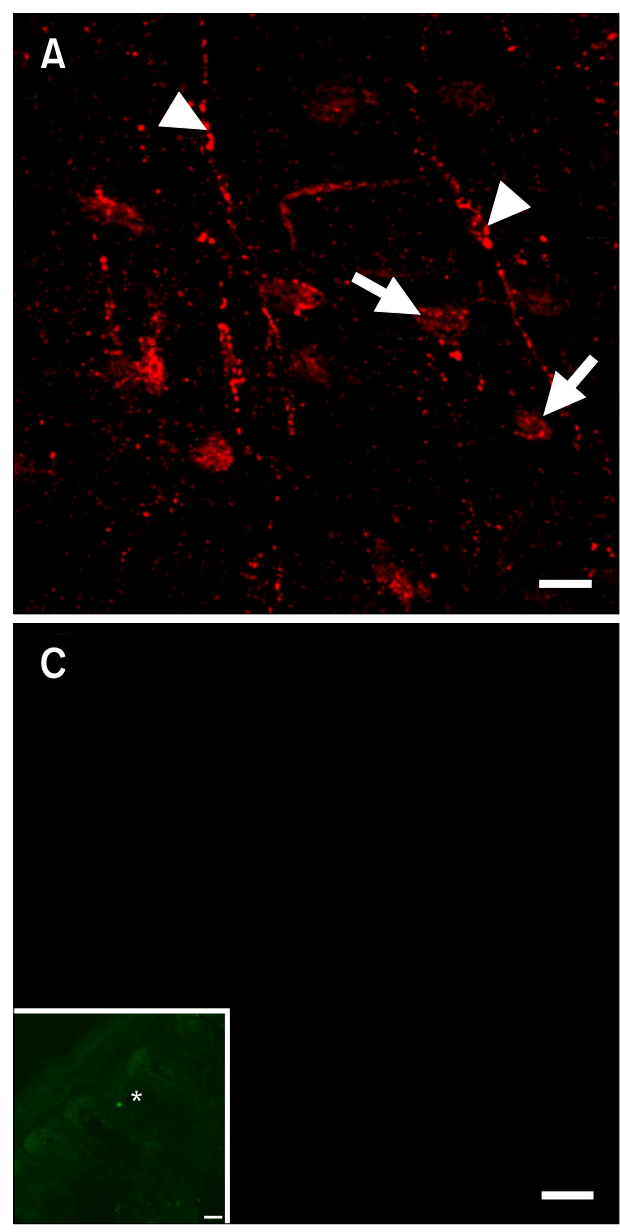

\section{B}

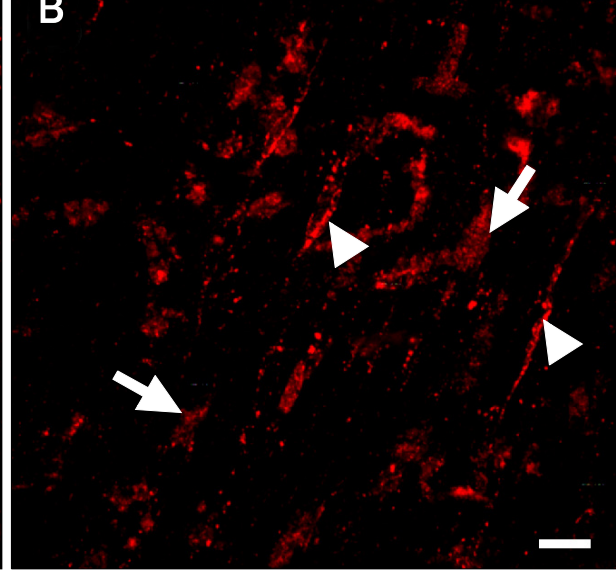

D

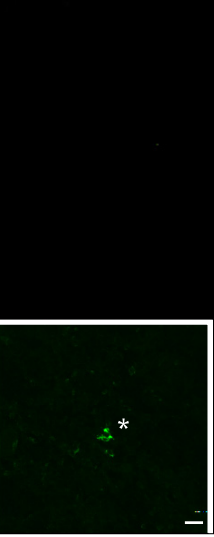

Figure 4. $\mathrm{Kit}^{+}$cells at the level of the myenteric plexus are interstitial cells of Cajal (ICC). (A and B) Development of $\mathrm{Kit}^{+} \mathrm{ICC}$ at the level of the myenteric plexus (arrows) in $W / W^{V}$ small intestine after transplantation of $\mathrm{C} 57 \mathrm{BL} / 6 \mathrm{BM}$, ICC associated with the deep muscular plexus are labeled with arrowheads. $\mathrm{Kit}^{+}$ ICC were not observed co-labeled with histamine (C) or F4/80 (D) within the same sections. Inserts demonstrate positive control staining of histamine (* in $\mathrm{C}$, inset) and $\mathrm{F} 4 / 80$ (* in $\mathrm{D}$, inset) antibodies in mouse small intestine cryostat sections. Scale bars $=20 \mu \mathrm{m}$. 
cells of $W / W^{V}$ muscles transplanted with BM cells from C57BL/6 mice were also more hyperpolarized, averaging $-59.3 \pm 1.3 \mathrm{mV}$ $(P=0.007, \mathrm{n}=4)$. Although RMP of transplanted intestines was similar to $\mathrm{Kit}^{+\operatorname{cop} G F P}$ (Fig. 1) and C57BL/6 intestinal tissues $^{27}$ slow wave activity was never observed. Although slow waves were absent from $W / W^{V}$ intestines transplanted with BMCs, RMP was significantly noisier than in intestinal muscles of non-transplanted $W / W^{V}$ mice. Noisy RMPs have been reported in various GI smooth muscle preparations and this activity has been termed unitary potentials, attributable to spontaneous inward current generated by ICC. ${ }^{32-34}$ An ongoing discharge of unitary potentials was recorded from intestinal muscles obtained from $W / W^{V}$ mice transplanted with BM cells from either $\mathrm{Kit}^{+/ \operatorname{cop} G F P}$ or C57BL/6 mice (Fig. 5). Unitary potential activity from intestines transplanted with BMCs from $\mathrm{Kit}^{+/ \operatorname{cop} G F P}$ and $\mathrm{C} 57 \mathrm{BL} / 6$ mice was quantified using a membrane potential probability density spread and compared with control $W / W^{V}$ intestines. Data were fitted with Gaussian curves to reveal differences in unitary potential activity. There were significant increases in the half maximal width of the membrane potential distribution in intestinal circular muscles of $W / W^{V}$ transplanted animals compared to non-transplanted tissues. Control non-transplanted $W / W^{V}$ muscles displayed a membrane potential distribution with a half maximum width of $0.83 \pm 0.13 \mathrm{mV}$ however $W / W^{V}$ tissues transplanted

\section{A}

$W / W^{\prime}$ control

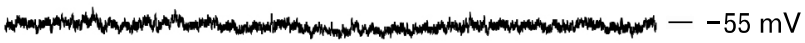

B

$W / W^{\prime}+C 57 B L / 6 B M$

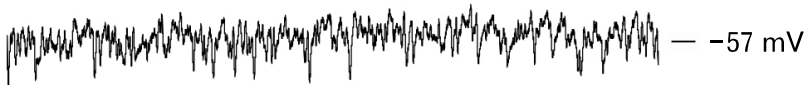

\section{C} $W / W^{\prime}+\mathrm{Kit}^{+/ \text {COPGFP }} \mathrm{BM}$

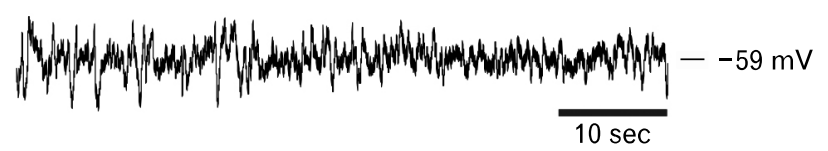

Figure 5. Development of unitary potential activity in bone marrow (BM) transplanted $W / W^{V}$ intestines. (A) Representative intracellular microelectrode recordings displaying electrical quiescence in $W / W^{V}$ control (non-transplanted) tissue. (B) Development of unitary potential activity in $W / W^{V}$ intestines following transplantation of $\mathrm{C} 57 \mathrm{BL} / 6$ derived bone marrow. (C) Shows unitary potential activity in $W / W^{V}$ intestines following transplantation of $\mathrm{Kit}^{+\operatorname{lop} G F P} \mathrm{BM}$ cells. with $\mathrm{C} 57 \mathrm{BL} / 6$ or $\mathrm{Kit}^{+/ \operatorname{cop} G F P}$ BMCs displayed distributions with a half maximum widths of $2.36 \pm 0.26 \mathrm{mV}(P=0.002, \mathrm{n}=4)$ and $2.1 \pm 0.28 \mathrm{mV}(P=0.006, \mathrm{n}=4$; Fig. 6$)$.

\section{Intestinal Mechanical Activity Following Bone Marrow Transplantation}

Motility patterns in intestines of $W / W^{V}$ mice were analyzed before and after transplantation of BMCs using STMaps constructed from imaging analysis of surface marker arrays (described in methods) placed on the submucosal surface of in-
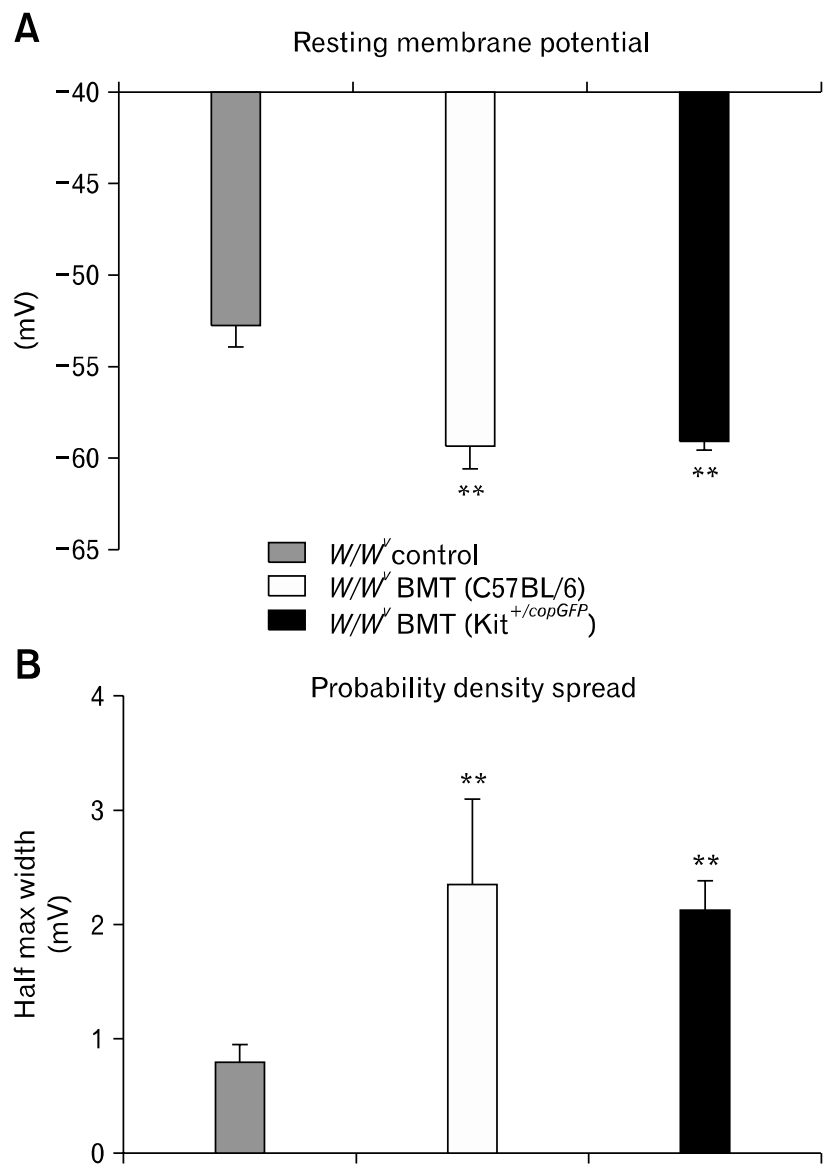

Figure 6. Summary of intracellular recordings revealing changes in resting membrane potential and probability density of unitary potential fluctuations. (A) Resting membrane potential was significantly different in $W / W^{V}$ tissues transplanted with C57BL/6 (white bars) or Kit ${ }^{+/ \operatorname{cop} G F P}$ (black bars) $\mathrm{BM}$ versus non-transplanted $W / W^{V}$ (grey bars). (B) Increases in the electrical activity were measured by fitting the probability density spread of unitary potential amplitude. The half max width of probability density spread was significantly increased upon transplantation of $\mathrm{Kit}^{+\operatorname{lop} G F P}$ (black bars) or C57BL/6 (white bars) BM versus non-transplanted $W / W^{V}$ (grey bars). BMT, bone marrow transplanted $\left({ }^{* *} P<0.01\right)$. 
testinal segments. STMaps of longitudinal movements in wildtype animals $(n=5)$ showed regular pendular movements in the longitudinal axis along the entire length of the preparation at a frequency of (about 20 cycles/min; Fig. 7A). Segmental contractions in the circular axis were masked by this longitudinal activity and were not easily resolved. In non-transplanted $W / W^{V}$ mice $(n=5)$, longitudinal movements of the ileum were detected, but the activity recorded was highly variable. Some regions of the preparation displayed contractile activity, but regions were often quiescent (Fig. 7B). A bursting pattern of activity was observed in $W / W^{V}$ intestines consisting of abrupt, large amplitude longitudinal contractions lasting about 5 seconds, followed by periods of quiescence (bottom maps in Fig. 7B), as previously described. $^{26}$ Intestines from $W / W^{V}$ mice transplanted with BM cells $(n=6)$ displayed abnormal patterns of movement similar to the non-transplanted $W / W^{V}$ intestines. Both bursting activity and irregular sites of initiation were observed (Fig. 7C). There was no consistent propagation of longitudinal contractions as observed in wild type intestinal muscles.
A
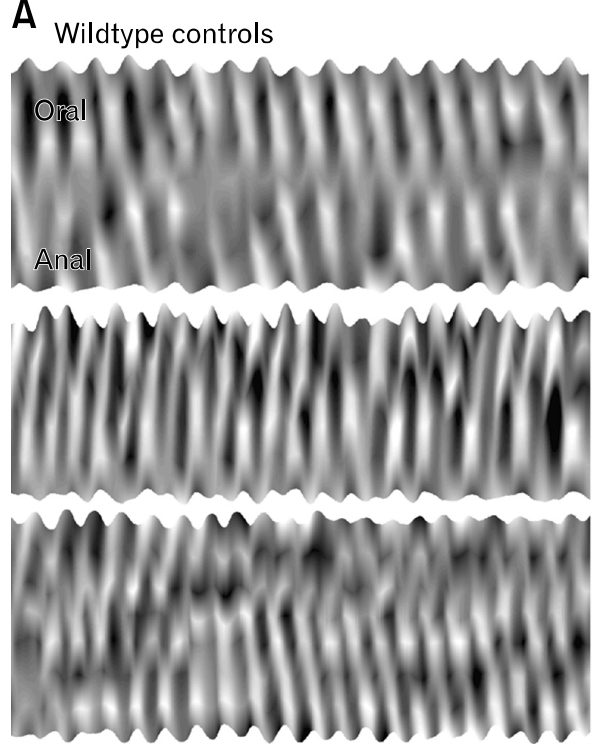

\section{B}
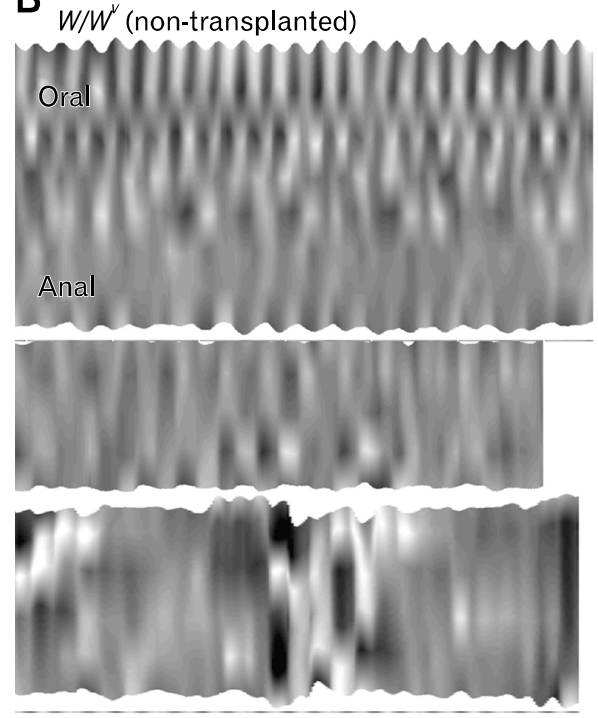

$-0.3+0.32 \mathrm{~mm}$

$5 \mathrm{sec}$
C $W / W^{*}+\mathrm{BMT}$
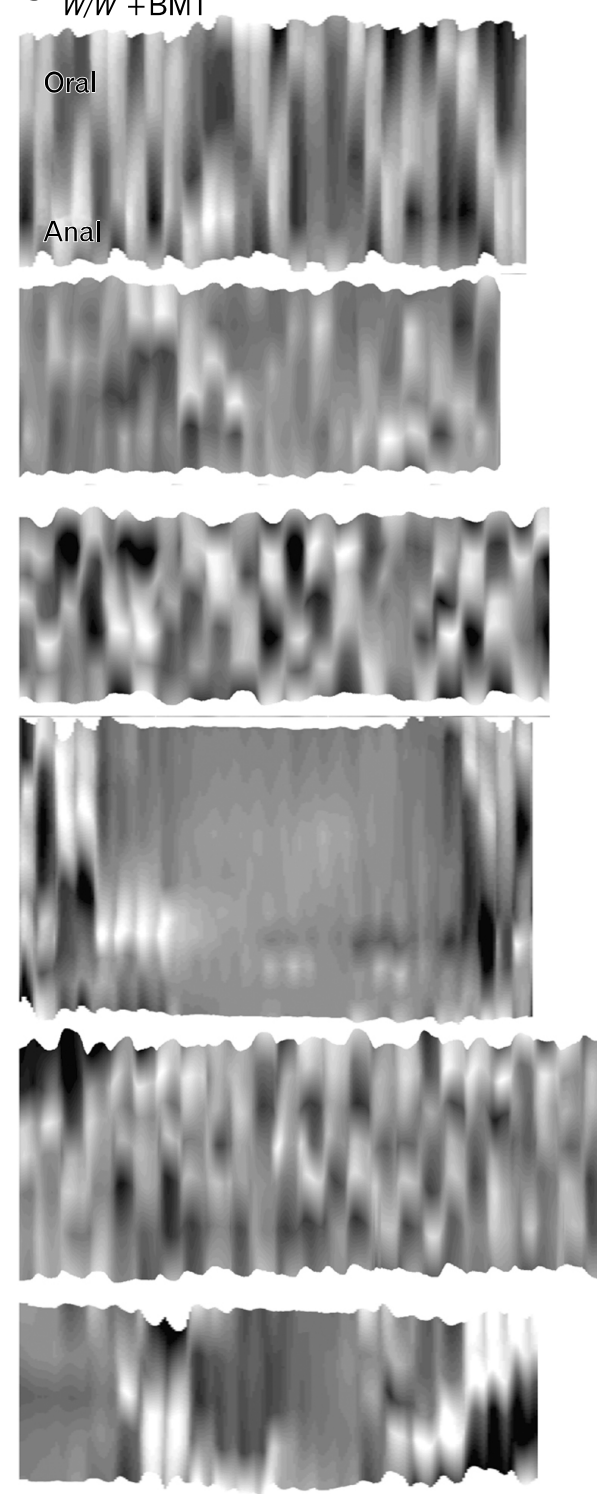

$-0.3+0.32 \mathrm{~mm}$

$5 \mathrm{sec}$
Figure 7. Bone marrow (BM) transplantation did not restore normal contractile activity. Representative spatiotemporal maps (STMaps) of longitudinal movement in the mouse ileum. (A) shows the representative pattern of movement in 3 wild type controls, consisting of regularly-occurring longitudinal contractions that propagated along the preparation. (B) In 3 representative $W / W^{V}$ mice, longitudinal contractions were observed in different regions along the preparation, and often occurred as a burst of activity for about 5 seconds followed by quiescence. There was no consistent propagation of longitudinal contractions. (C) Shows longitudinal movements in $6 \mathrm{BM}$ transplanted (BMT) $W / W^{V}$ mice, which closely resembled the pattern of activity in $W / W^{V}$ without BMT. Variable sites of initiation, abrupt contractions and bursting activity followed by quiescence were observed. No consistent propagation of longitudinal contractions was observed. All STMaps have the same scaling of length (vertical axis), time (horizontal axis) and amplitude (grayscale - see methods for explanation). 


\section{Discussion}

ICC perform several essential physiological roles in GI motility and the loss or disruption of ICC networks in patients with a number of diverse motor disorders supports the hypothesis that they provide fundamental regulatory control of GI motility. ${ }^{35}$ $\mathrm{Kit} / \mathrm{stem}$ cell factor (SCF) signaling is essential for the development and maintenance of functional ICC networks. ${ }^{36}$ Chemical lesion of the Kit signaling pathway through administration of the neutralizing antibody $\mathrm{ACK} 2^{37-39}$ or the receptor antagonist Imatinib or Gleevec ${ }^{36}$ leads to a loss of ICC.

Mutations in the Kit receptor, as occurs in the compound heterozygote $W / W^{V}$ mouse also leads to disruption of ICC networks at the level of the myenteric plexus in the small intestine and a loss of slow wave activity. ${ }^{27,28}$ Although Kit signaling is disrupted in $W / W^{V}$ mutants, SCF expression appears to be normal (Ward and Hwang, unpublished observations). Therefore the intestines of $W / W^{V}$ mutant mice provide an excellent cellular and physiological model for transplantation studies of ICC into tissues normally devoid of these cells.

Several recent studies have examined the feasibility of incorporation of BM derived cells into the GI tracts of several models with the aim to re-populate tissues that have lost ICC following intestinal injury, ${ }^{23}$ or are naturally devoid of ICC as occurs in Kit deficient mice. Using immunohistochemical analysis, these studies reported that BM derived cells which were $\mathrm{Kit}^{+}{ }^{\text {in- }}$ corporated into the intestine at the level of the myenteric and deep muscular plexi. ${ }^{24}$ However, although one of these studies reported slightly improved intestinal motility and transit time in $W / W^{V}$ mutants following transplantation, no other functional studies were performed to determine if the incorporated cells develop full functionality. ${ }^{24}$ Evidence was not provided whether the $\mathrm{BM}$ derived $\mathrm{Kit}^{+}$cells formed discrete networks typical of ICC-MY, or were capable of generating pacemaker activity.

In the present study we explored the possibility of restoring ICC using BM derived cells marked with a Kit-linked reporter. We performed immunohistochemical and Western analysis to demonstrate the incorporation of BM derived $\mathrm{Kit}^{+}$cells into the small intestines of $W / W^{V}$ mice, as previously reported. ${ }^{23,24}$ BMCs, isolated from Kit ${ }^{+\operatorname{cop} G F P}$ mice (Fig. 2), a model in which $\mathrm{Kit}^{+}$ cells express endogenous copGFP, were used in a series of transplantation studies to allow us to track the development of ICC in $W / W^{V}$ mice. We aimed to replicate the clinical application of a therapeutic intervention with minimal treatment of $\mathrm{BMCs}$ prior to transplantation using a well established BM transplantation protocol (see reference 25). This was slightly different to a previously studies in which BMCs were supplemented with media and fetal bovine serum. In the current study the identification of $\mathrm{Kit}^{+\operatorname{cop} G F P}$ positive BM marrow derived cells 12 weeks post transplantation, provides evidence that the cells were viable and the method of transplantation robust.

Although $\mathrm{Kit}^{+}$-cells incorporated into the intestines of $W / W^{V}$ mutants 12 weeks after transplantation, they did not form anastomosing networks which interconnect with each other, typical of intestinal ICC-MY. ${ }^{27}$ Whilst $\mathrm{Kit}^{+}$cells, that were negative for mast cell (histamine) and macrophage (F4/80) markers, were occasionally observed in clusters they usually possessed a triangular shape and remained in relative isolation to one another. No network forming projections were observed to emanate from their cell bodies as revealed by whole mounts. Western analysis of $W / W^{V}$ intestinal tissues 12 weeks following $\mathrm{BM}$ transplantation highlighted an increase in Kit protein expression compared with control. The size of Kit protein has previously been shown to vary. ${ }^{40}$ This is possibly due to the existence of different splicing isoforms or potential glycosylation sites. ${ }^{41}$ Within this transplantation assay we detected 3 major bands corresponding to Kit protein (Fig. 3) at approximately $140 \mathrm{kDa},{ }^{42} 125 \mathrm{kDa}^{43}$ and 80 $\mathrm{kDa}^{44}$

To determine the functional characteristics of BM derived $\mathrm{Kit}^{+}$cells in transplanted intestines we performed intracellular microelectrode recordings and surface marker arrays to determine coordination of spontaneous contractile activity. Control tissues had relatively hyperpolarized membrane potentials $(-60$ $\mathrm{mV}$ ) and generated regular slow waves. Associated with this spontaneous pacemaker activity were regular pendular movements of the intestinal tunica muscularis. In comparison, membrane potential of control $W / W^{V}$ intestines were more depolarized and did not generate slow waves. The presence of BM derived $\mathrm{Kit}^{+}$cells transplanted into the intestines of $W / W^{V}$ mice led to a return in membrane potential, similar to that observed in control mice with normal ICC networks. ${ }^{27}$ Although membrane potential was similar to that of control intestines slow wave activity did not develop in any of the tissues examined after the 12-week transplantation period. Nevertheless, unlike control $W / W^{V}$ intestines which display electrical quiescence, $\mathrm{BM}$ transplantation led to a significant increase in small amplitude fluctuations in membrane potential termed 'unitary potentials.' It has been demonstrated that ICC in different regions of the GI tract possess the capability to generate unitary potentials that contribute to the overall excit- 
ability of GI muscles. ${ }^{45,46}$ It has been further suggested that summation in the stochastic discharge of unitary potentials leads to the generation of pacemaker potentials resulting in slow wave activity. ${ }^{33}$ The development of unitary potential like fluctuations together with $\mathrm{Kit}^{+}$phenotype and location of BM derived cells within the myenteric plexus region suggested that these cells were ICC which lack the ability to send out projections and form a fully functional network.

The results of the present study are in contrast to previous reports $^{24}$ as BM transplantation did not appear to have beneficial effects on contraction coordination as examined with surface marker arrays. The failure of BM derived cells to form anastomosing ICC networks at the level of myenteric plexus and the accompanying failure to elicit slow wave pacemaker potentials may be responsible for the observed contractile behavior in BM transplanted preparations. However, it remains unclear if the increase in unitary potential activity described in this study could be responsible for the slight increase in transit time highlighted in a previous study. ${ }^{24}$ Also the relative rarity of BM derived ICC incorporation into the deep muscular plexus, where ICC-DMP are present in $W / W^{V}$ mutant mice, may suggest a space limiting factor in the extravasation of $\mathrm{BM}$ to the tunica muscularis. Loss of ICC via genetic deficiency or pathological insult of the GI tract might allow for incorporation of ICC, whereas during normal development BM modulation of ICC numbers may not necessarily occur.

The mechanisms of maintenance and turnover of ICC, in vivo, remain controversial, however it is well recognized that ICC display a high degree of plasticity and regenerative capacity. ICC restoration has been observed in a variety of models including chemical lesion, ${ }^{36,39}$ surgical lesion ${ }^{15,16}$ and inflammation. ${ }^{47}$ In these studies ICC networks were disrupted following the pathophysiological insult but restoration of functional ICC networks occurred upon removal of the insult.

Although ICC possess a degree of plasticity, the mechanism of how they repopulate GI tissues is controversial. It has been shown that ICC undergo a phenotypic change and adopt a smooth muscle phenotype, including expression of thick filaments in response to disruption of the Kit signaling pathway. ${ }^{10}$ Others have recently reported that several cell phenotypes including ICC, within the tunica muscularis undergo apoptosis in response to ischemic reperfusion of the small intestine and subsequent proliferation was involved in their recovery. ${ }^{12}$ It has also been shown that ICC undergo apoptosis in healthy colon as a natural process to regulate numbers that must continually regenerate to maintain functional networks. ${ }^{48}$

Recently a population of presumed ICC progenitor/stem cells has been identified in murine gastric muscles. These progenitor cells display a $\mathrm{Kit}^{\text {low }} \mathrm{CD} 44{ }^{+} \mathrm{CD} 34{ }^{+} \mathrm{Insr}^{+} \mathrm{Igflr}^{+}$phenotype and have been postulated to be involved in the constant remodeling of ICC networks in the stomach. ${ }^{13}$ Within this study BM derived cells presented as $\mathrm{Kit}^{+}$suggesting that they are more mature than the progenitor cells described above.

In summary, we have highlighted the failure of BM derived cells to form functional ICC networks and restore pacemaker function in $W / W^{V}$ mice that naturally lack ICC-MY. It remains unclear why BM derived cells have the ability to, extravasate from the BM, incorporate within the tunica muscularis of the small intestine and generate unitary potentials but fail to form fully functional networks capable of generating slow wave activity. Further studies are required to elucidate the mechanisms, together with the genetic and microenvironmental factors, involved in the development and patterning of fully functional ICC networks. Such studies may provide evidence as to why BM derived cells fail to form functional networks. Based on the present findings, BM transplantation may not provide a viable mechanism of restoring ICC networks in patients displaying pathological loss of ICC and GI dysmotility.

\section{Acknowledgements}

The authors would like to acknowledge the excellent technical support provided by Yulia Bayguinov.

\section{References}

1. Gockel I, Bohl JR, Eckardt VF, Junginger T. Reduction of interstitial cells of Cajal (ICC) associated with neuronal nitric oxide synthase (n-NOS) in patients with achalasia. Am J Gastroenterol 2008; 103:856-864.

2. Kenny SE, Vanderwinden JM, Rintala RJ, et al. Delayed maturation of the interstitial cells of Cajal: a new diagnosis for transient neonatal pseudoobstruction. Report of two cases. J Pediatr Surg 1998;33:9498.

3. Isozaki K, Hirota S, Miyagawa J, Taniguchi M, Shinomura Y, Matsuzawa Y. Deficiency of c-kit ${ }^{+}$cells in patients with a myopathic form of chronic idiopathic intestinal pseudo-obstruction. Am J Gastroenterol 1997;92:332-334

4. Porcher C, Baldo M, Henry M, Orsoni P, Julé Y, Ward SM. Deficiency of interstitial cells of Cajal in the small intestine of patients with Crohn's disease. Am J Gastroenterol 2002;97:118-125.

5. Rumessen JJ. Ultrastructure of interstitial cells of Cajal at the colonic submuscular border in patients with ulcerative colitis. Gastroenterol- 
ogy 1996;111:1447-1455.

6. Ordög T, Takayama I, Cheung WK, Ward SM, Sanders KM. Remodeling of networks of interstitial cells of Cajal in a murine model of diabetic gastroparesis. Diabetes 2000;49:1731-1739.

7. Wang XY, Huizinga JD, Diamond J, Liu LW. Loss of intramuscular and submuscular interstitial cells of Cajal and associated enteric nerves is related to decreased gastric emptying in streptozotocin-induced diabetes. Neurogastroenterol Motil 2009;21:1095, e92.

8. Torihashi S, Ward SM, Sanders KM. Development of c-Kit-positive cells and the onset of electrical rhythmicity in murine small intestine. Gastroenterology 1997;112:144-155.

9. Young HM, Ciampoli D, Southwell BR, Newgreen DF. Origin of interstitial cells of Cajal in the mouse intestine. Dev Biol 1996;180: 97-107.

10. Torihashi S, Nishi K, Tokutomi Y, Nishi T, Ward S, Sanders KM. Blockade of kit signaling induces transdifferentiation of interstitial cells of cajal to a smooth muscle phenotype. Gastroenterology 1999; 117:140-148.

11. Mei F, Han J, Huang Y, Jiang ZY, Xiong CJ, Zhou DS. Plasticity of interstitial cells of cajal: a study in the small intestine of adult Guinea pigs. Anat Rec(Hoboken) 2009;292:985-993.

12. Mei F, Guo S, He YT, et al. Apoptosis of interstitial cells of Cajal, smooth muscle cells, and enteric neurons induced by intestinal ischemia and reperfusion injury in adult guinea pigs. Virchows Arch 2009;454:401-409.

13. Lorincz A, Redelman D, Horvath VJ, Bardsley MR, Chen H, Ordög T. Progenitors of interstitial cells of cajal in the postnatal murine stomach. Gastroenterology 2008;134:1083-1093.

14. Han J, Zhou YP, Jiang YZ, He YT, Mei F. Postnatal development of interstitial cells of Cajal in mouse colon in response to Kit signal blockade with Imatinib (Glivec). Acta Histochem 2010;112:215221.

15. Yanagida H, Yanase H, Sanders KM, Ward SM. Intestinal surgical resection disrupts electrical rhythmicity, neural responses, and interstitial cell networks. Gastroenterology 2004;127:1748-1759.

16. Chang IY, Glasgow NJ, Takayama I, Horiguchi K, Sanders KM, Ward SM. Loss of interstitial cells of Cajal and development of electrical dysfunction in murine small bowel obstruction. J Physiol 2001; 536(Pt 2):5 55-568.

17. Braga LM, Rosa K, Rodrigues B, et al. Systemic delivery of adult stem cells improves cardiac function in spontaneously hypertensive rats. Clin Exp Pharmacol Physiol 2008;35:113-119.

18. Zhang S, Jia Z, Ge J, et al. Purified human bone marrow multipotent mesenchymal stem cells regenerate infarcted myocardium in experimental rats. Cell Transplant 2005;14:787-798.

19. Chamberlain J, Yamagami T, Colletti E, et al. Efficient generation of human hepatocytes by the intrahepatic delivery of clonal human mesenchymal stem cells in fetal sheep. Hepatology 2007;46:1935-1945.

20. Almeida-Porada G, Porada CD, Chamberlain J, Torabi A, Zanjani ED. Formation of human hepatocytes by human hematopoietic stem cells in sheep. Blood 2004;104:2582-2590.

21. Urbán VS, Kiss J, Kovács J, et al. Mesenchymal stem cells cooperate with bone marrow cells in therapy of diabetes. Stem Cells 2008;26: 244-253.

22. Rojas $\mathrm{M}, \mathrm{Xu} \mathrm{J}$, Woods $\mathrm{CR}$, et al. Bone marrow-derived mesenchymal stem cells in repair of the injured lung. Am J Respir Cell Mol
Biol 2005;33:145-152.

23. Liu D, Wang F, Zou Z, et al. Bone marrow derivation of interstitial cells of cajal in small intestine following intestinal injury. J Biomed Biotechnol 2010;2010:164986.

24. Ishii S, Tsuji S, Tsujii M, et al. Restoration of gut motility in Kit-deficient mice by bone marrow transplantation. J Gastroenterol 2009; 44:834-841.

25. Sun K, Li M, Sayers TJ, Welniak LA, Murphy WJ. Differential effects of donor T-cell cytokines on outcome with continuous bortezomib administration after allogeneic bone marrow transplantation. Blood 2008;112:1522-1529.

26. Hennig GW, Costa M, Chen BN, Brookes SJ. Quantitative analysis of peristalsis in the guinea-pig small intestine using spatio-temporal maps. J Physiol 1999;517(Pt 2):575-590.

27. Ward SM, Burns AJ, Torihashi S, Sanders KM. Mutation of the proto-oncogene c-kit blocks development of interstitial cells and electrical rhythmicity in murine intestine. J Physiol 1994;480(Pt 1):9197.

28. Huizinga JD, Thuneberg L, Klüppel M, Malysz J, Mikkelsen HB, Bernstein A. W/kit gene required for interstitial cells of Cajal and for intestinal pacemaker activity. Nature 1995;373:347-349.

29. Ro S, Park C, Jin J, et al. A model to study the phenotypic changes of interstitial cells of Cajal in gastrointestinal diseases. Gastroenterology 2010;138:1068-1078, e2.

30. Li Y, Kojima H, Fujino K, et al. Homing of the Bone MarrowDerived Interstitial Cells of Cajal is Decreased in Diabetic Mouse Intestine. J Gastroenterol Hepatol 2011;26:1072-1078.

31. Ozaki H, Kawai T, Shuttleworth CW, et al. Isolation and characterization of resident macrophages from the smooth muscle layers of murine small intestine. Neurogastroenterol Motil 2004;16:39-51.

32. Burns AJ, Lomax AE, Torihashi S, Sanders KM, Ward SM. Interstitial cells of Cajal mediate inhibitory neurotransmission in the stomach. Proc Natl Acad Sci USA 1996;93:12008-12013.

33. Hirst GD, Edwards FR. Generation of slow waves in the antral region of guinea-pig stomach--a stochastic process. J Physiol 2001; 535(Pt 1):165-180.

34. Beckett EA, Bayguinov YR, Sanders KM, Ward SM, Hirst GD. Properties of unitary potentials generated by intramuscular interstitial cells of Cajal in the murine and guinea-pig gastric fundus. J Physiol 2004;559(Pt 1):259-269.

35. Burns AJ. Disorders of interstitial cells of Cajal. J Pediatr Gastroenterol Nutr 2007;45(suppl 2):S103-106.

36. Beckett EA, Ro S, Bayguinov Y, Sanders KM, Ward SM. Kit signaling is essential for development and maintenance of interstitial cells of Cajal and electrical rhythmicity in the embryonic gastrointestinal tract. Dev Dyn 2007;236:60-72.

37. Maeda H, Yamagata A, Nishikawa S, et al. Requirement of c-kit for development of intestinal pacemaker system. Development 1992;116: 369-375.

38. Torihashi S, Ward SM, Nishikawa S, Nishi K, Kobayashi S, Sanders KM. c-kit-dependent development of interstitial cells and electrical activity in the murine gastrointestinal tract. Cell Tissue Res 1995; 280:97-111.

39. Ward SM, Harney SC, Bayguinov JR, McLaren GJ, Sanders KM. Development of electrical rhythmicity in the murine gastrointestinal tract is specifically encoded in the tunica muscularis. J Physiol 
1997;505(Pt 1):241-258

40. Majumder S, Brown K, Qiu FH, Besmer P. c-kit protein, a transmembrane kinase: identification in tissues and characterization. Mol Cell Biol 1988;8:4896-4903.

41. Lyman SD, Jacobsen SE. c-kit ligand and Flt3 ligand: stem/progenitor cell factors with overlapping yet distinct activities. Blood 1998;91: 1101-1134.

42. Wakayama T, Kato Y, Utsumi R, Tsuji A, Iseki S. A time- and cost-saving method of producing rat polyclonal antibodies. Acta Histochem Cytochem 2006;39:79-87.

43. Tonary AM, Macdonald EA, Faught W, Senterman MK, Vanderhyden BC. Lack of expression of c-KIT in ovarian cancers is associated with poor prognosis. Int J Cancer 2000;89:242-250.

44. Besmer P, Lader E, George PC, et al. A new acute transforming feline retrovirus with fms homology specifies a C-terminally truncated version of the c-fms protein that is different from SM-feline sarcoma virus v-fms protein. J Virol 1986;60:194-203.

45. Dickens EJ, Hirst GD, Tomita T. Identification of rhythmically active cells in guinea-pig stomach. J Physiol 1999;514(Pt 2):515-531.

46. Kito Y, Suzuki H, Edwards FR. Properties of unitary potentials recorded from myenteric interstitial cells of Cajal distributed in the guinea-pig gastric antrum. J Smooth Muscle Res 2002;38:165-179.

47. Wang XY, Vannucchi MG, Nieuwmeyer F, Ye J, FaussonePellegrini MS, Huizinga JD. Changes in interstitial cells of Cajal at the deep muscular plexus are associated with loss of distention-induced burst-type muscle activity in mice infected by Trichinella spiralis. Am J Pathol 2005;167:437-453.

48. Gibbons SJ, De Giorgio R, Faussone Pellegrini MS, et al. Apoptotic cell death of human interstitial cells of Cajal. Neurogastroenterol Motil 2009;21:85-93. 